\title{
Die Entstehung der dänischen und norddeutschen Rinnentäler (Tunneltäler) - Glaziologische Gesichtspunkte
}

\author{
PER SMED*) \\ rinnen valley, tunnel valley, wide tunnels, law of ice movement, law of water movement, \\ buried valleys, glacier tongue theory
}

\begin{abstract}
Kurzfassung: Die alte Tunneltal-Theorie (Ussing 1907) ist immer umstritten gewesen, besonders weil die so benannten Täler mehr als $1 \mathrm{~km}$ breit sein können. Es wurde behauptet, daß so breite Tunnel oder Hohlräume unter Gletschereis unmöglich seien. Seit 1950 haben Fortschritte in der Glaziologie (besonders NyE 1952 und SHREve 1972) bewirkt, daß die Tunneltal-Idee neu belebt worden ist. Lange und schmale Eiszungen, wie sie noch heute auf vielen Karten von Norddeutschland gezeigt werden, sind nach NyE nur in Gebirgslandschaften möglich. Dagegen entspricht SHreves Bild vom Verlauf subglazialer Wasserströme genau dem aus Dänemark, Holstein und Mecklenburg bekannten Muster von Osern und Rinnentälern. Kuster \& MEYER (1979) und EHLERS \& LINKE (1989) interpretieren die begrabenen norddeutschen Täler (,incisions“) als subglaziale Schmelzwasserrinnen. Bis heute fehlt jedoch eine befriedigende Erklärung der zuweilen großen Talbreite. Eine Gruppe dänischer Forscher (vor allem HumLum) hat versucht, diesem Mangel abzuhelfen. Es ergibt sich, daß ein sukzessives Entstehen die wahrscheinlichste Lösung ist. Im Winter, wenn wenig Schmelzwasser anfällt, preßt das Gletschereis den Tunnel zusammen, so daß der Tunnelquerschnitt die verstärkte Wasserführung des Frühjahrs nicht fassen kann. Ein neuer Tunnel muß in den Untergrund eingeschnitten werden. Wenn sich dieser Prozeß jedes Jahr wiederholt, ist das Resultat eine breite Talsohle. Es wird gezeigt, daß die Morphologie dänischer Tunneltäler bis in alle Einzelheiten durch diesen Vorgang erklärt werden kann, und daß eine entsprechend detaillierte Erklärung durch keine der bis heute angebotenen alternativen Theorien geboten werden kann.
\end{abstract}

\section{[The genesis of the so-called "rinnen" incisions (tunnel valleys) in Denmark and North Ger- many. A glaciological approach.]}

\begin{abstract}
The original tunnel valley theory was put forward by Ussing 1907. Its validity has, however, been doubted by most German geologists since then, especially because many so-called tunnel valleys are surprisingly wide ( $1 \mathrm{~km}$ or more). It was claimed that so wide tunnels or cavities under the ice were impossible. Since 1950, progress in glaciology (especially NyE 1952 and SHREve 1972) has caused a revival of the tunnel valley
\end{abstract}

* P. SMED, Lektor, cand. mag., Stiholmsvej 1, 8, DK-3460 Birkerød, Dänemark concept. Long and narrow ice tongues which have been postulated for North Germany by some researchers, according to NYE are only possible in mountainous terrain. On the other hand, SHREve's picture of the subglacial stream pattern matches precisely the known pattern of eskers and valley incisions in Denmark, Holstein, and Mecklenburg. Kuster \& MeyER (1979) and EHLERs \& LINKE (1989) interpret the buried valley incisions in northern Germany as eroded by subglacial streams. Until now, however, their large width has not been sufficiently explained. A group of Danish scientists including Ole Humlum has discussed this problem. A successive origin of the valleys appears to be most probable: during the winter with its reduced meltwater flow, the tunnel is narrowed by ice pressure. Therefore, it cannot carry the vastly increased amount of water during the spring, and a new tunnel has to be eroded, presumably partly into the substratum. Annual repetition of this process causes wide valley bottoms, only small parts of which were ever water-filled at any given time. It is shown that the morphology of the Danish tunnel valleys can be explained in every detail by such a sequence of events, and that no alternative theory can offer an equally satisfactory explanation.

Vor knapp 100 Jahren hat der dänische Geologe N.V. Ussing $(1903,1907)$ die Auffassung vertreten, daß die großen Täler in Mitteljütland durch Schmelzwassererosion unter dem Eis entstanden seien. Obwohl Täler desselben Typs in der südlichen Umgebung der Ostsee weit verbreitet sind, regten sich in Deutschland bald skeptische Stimmen. Es schien unvorstellbar, daß durch subglaziale Schmelzwässer Täler von solcher Breite entstehen könnten. KaRL GrIPP wies darauf hin, daß die Flensburger Förde $3 \mathrm{~km}$ breit sei, der Aabenraa Fjord sogar 4 km. Für GRIPP (1964) und PAUL Woldstedt (1950) stand fest, daß diese Rinnen von schmalen Gletscherzungen ausgeschürft worden sein mußten, wie man sie von den Alpen her kennt.

Man wußte jedoch seinerzeit recht wenig davon, was im und unter dem Eis vorging. In der Folge der Untersuchungen von "Camp-Century" auf 


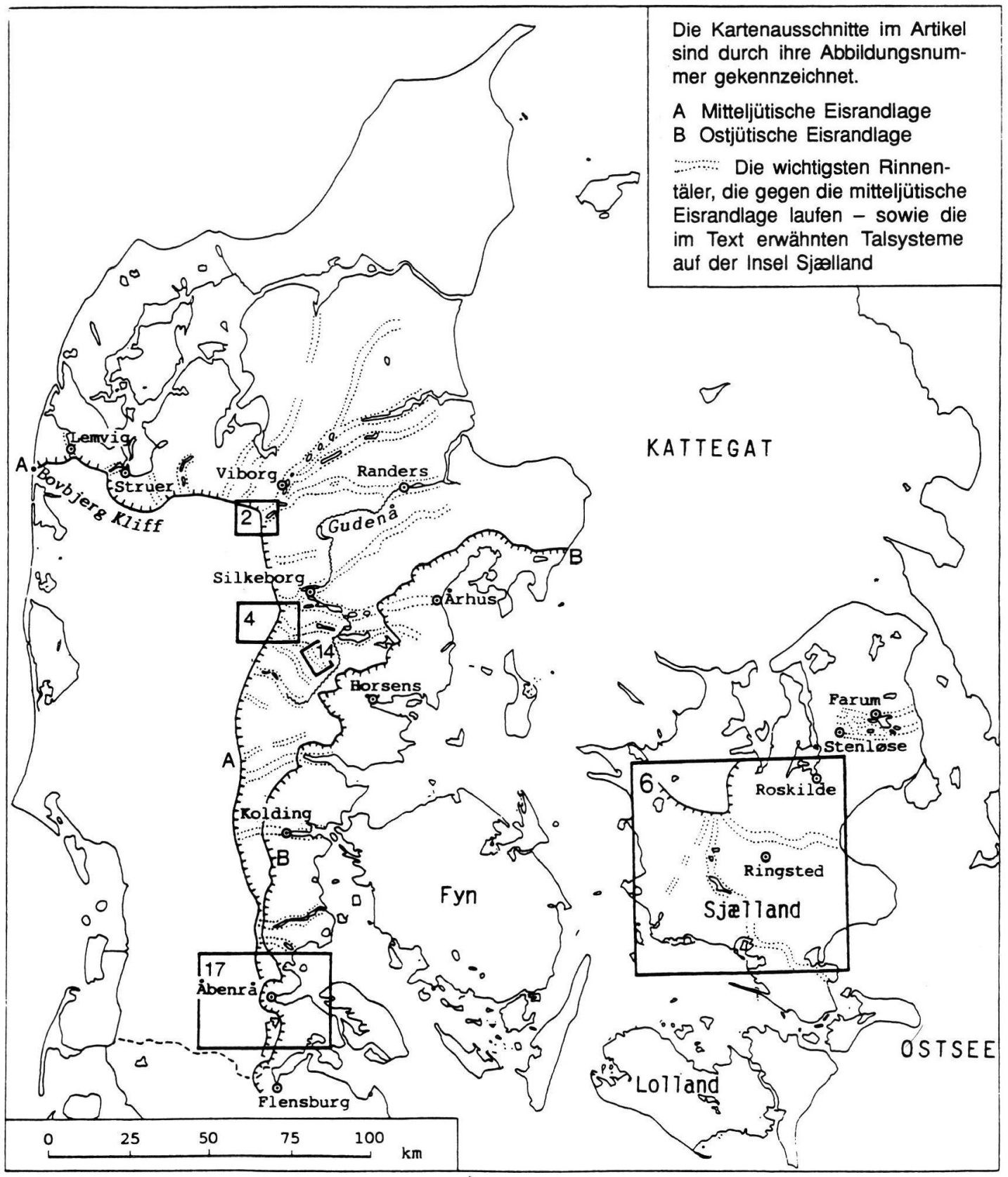

Abb 1: Lage der im Artikel erwähnten Lokalitäten.

Fig. 1: Localities mentioned in the article. Map sections used as illustrations are designated through their figure numbers. A: Mid Jutland ice margin line. B: East Jutland ice margin line. The most important "Rinnen" incisions in Jutland and on the island Sjælland are shown as dotted double lines.

dem nordgrönländischen Inlandeis bei Qanaq kam es in den 50er Jahren zu einem Aufblühen der Glaziologie. J. F. NYE (1952) arbeitete mit vielen zusammen daran, die Kräfte, die die Gletscherbewegung bewirken, in mathematischen Formeln auszudrücken. Es hat leider lange Zeit gebraucht, bis dieses Spezialwissen in die glazialmorphologischen und -geologischen Überlegungen in Dänemark, Schweden und Deutschland Eingang gefunden hat.

Aus den Formeln von NyE kann hergeleitet werden, daß in einem Tiefland wie Schleswig-Hol- 
stein, Mecklenburg oder Dänemark eine Gletscherzunge nur etwa halb so lang wie breit sein kann. Ihre Form kann daher nicht wesentlich länglicher als ein Halbkreis sein. In gebirgigem Gelände verhindern die steilen Talflanken, daß sich die Eiszungen nach allen Seiten gleichmäßig ausdehnen; letzteres ist aber der Fall, wenn keine seitliche Begrenzung vorliegt. Das kann man an heutigen Eiskappen in ebenem Gelände beobachten, z. B. den Nordrändern des Vatnajökull und des Myrdalsjökull auf Island. Gletscherzungen von 30-40 km Länge und 2-6 km Breite, wie man sie sich für die Entstehung der Tunneltäler vorgestellt hat, sind physikalisch in einer Landschaft wie der norddeutschen nicht möglich. Hinzu kommt, daß die gedachten Eiszungen sich aufwärts bewegt haben sollen, von der Ostsee und vom Kattegat her kommend bis in die Mitte von Jütland oder Mecklenburg. Das steht im Widerspruch zu den physikalischen Grundregeln. Die diesbezüglichen Vorstellungen GRIPPS über die eiszeitliche Formung Schleswig-Holsteins müssen aufgegeben werden.

In Dänemark ging die Tunneltal-Theorie in die Schulbücher ein und wurde auf diese Weise für mehrere Generationen zum Allgemeingut. Die
Skeptiker setzten sich dennoch am Ende durch (Kaj Hansen, 1971). Seine Schlußfolgerung, daß anstelle der Schmelzwassertunnel schmale Eiszungen die Täler geformt hätten, stimmte mit GRIPP überein. In den folgenden Jahren wurden viele Versuche unternommen, alternative Erklärungen zu finden.

Ussings $(1903,1907)$ Argumentation kann auf die folgende Weise dargestellt werden.

1. Die westjütischen Sanderflächen sind Schwemmkegel, entstanden, als das Schmelzwasser aus dem Gletschertor ins Freie trat. Der Strom teilte sich dort in viele schmale Arme auf, die sich fächerförmig nach allen Seiten ausbreiteten (Abb. 5 und 15). Das Ergebnis ist, daß ein flacher Sedimentkegel aufgeschüttet wird, dessen höchster Punkt am Gletschertor liegt. Alle westjütischen Sanderkegel haben ihren höchsten Punkt an der mitteljütischen „Hauptstillstandslinie“ der Weichselvereisung. Die großen ostjütischen Täler laufen rechtwinklig zur „Hauptstillstandslinie“, und die meisten von ihnen enden am höchsten Punkt eines angrenzenden Sanderkegels - nach UsSING somit am Gletschertor.

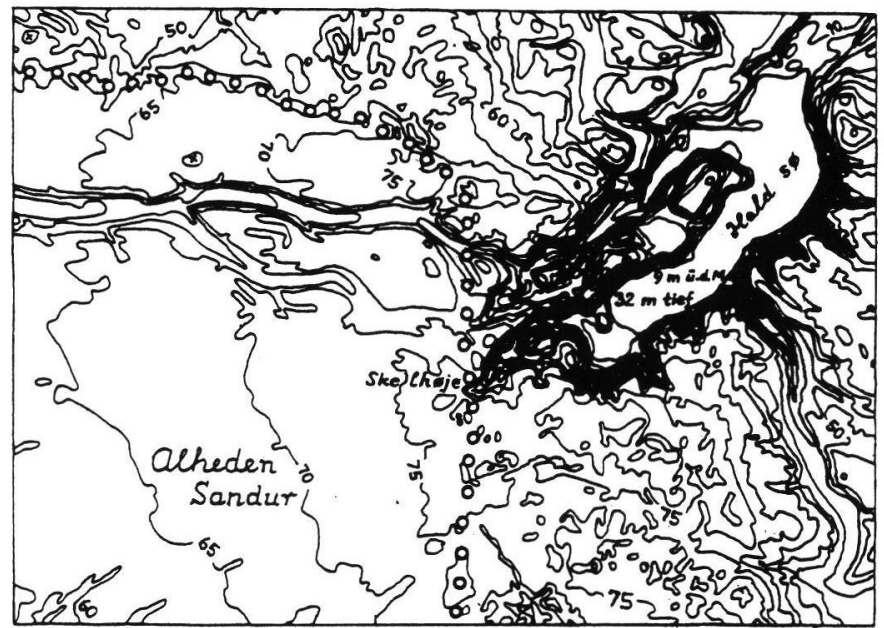

\begin{tabular}{lllll}
\hline & 1 & 1 & &
\end{tabular}

Äquidistanz $5 \mathrm{~m}$

\section{○०००० Mitteljütische Eisrandlage}

Abb. 2: Beispiel eines Tunneltales: Hald Sø (Hald-See) und Alheden Sandur, SW von Viborg, Jütland. Vervielfältigung mit Genehmigung Nr. A 246-96 von Kort- und Matrikelstyrelsen. Kopenhagen.

Fig. 2: Contour map of the transition between the Hald $S \varnothing$ incision (tunnel valley) and the Alheden sandur plain, SW of Viborg, Jutland. Contour interval 5 meters. The Mid Jutland ice margin line is shown as a row of small circles.

Die Isohypsendarstellung sowohl in Abb. 2 als auch in Abb. 4 (oben) zeigt das westliche Ende eines der großen ostjütischen Talsysteme sowie einen Teil des vorgelagerten Schmelzwassersanders. Die konzentrisch verlaufenden Höhenlinien deuten die Kegelform des Sanders an (ein etwas jüngerer Schmelzwasserstrom hat sich in die Fläche eingeschnitten und dabei das heutige Kolpendal geformt). In Abb. 4 (unten) sind die Verhältnisse in einer geomorphologischen Kartenskizze erläutert. Man sieht, daß der SaltenåTalboden von einer Anzahl 20-30 m hoher Rücken überragt wird, und daß sich das Tal an seinem westlichen Ende in drei Arme aufspaltet. Die Talsohle steigt an der Übergangsstelle zum Schwemmkegel auf gut $100 \mathrm{~m}$ über dem Meeresspiegel an. Es liegt nahe, daß die drei "Finger" zu drei Gletschertoren geführt haben, die vielleicht nacheinander aktiv waren. Hieraus folgert, daß das Tal der heutigen Saltenå ( $2 \mathrm{~km}$ breit, $100 \mathrm{~m}$ tief) von Schmelzwasser aus- 


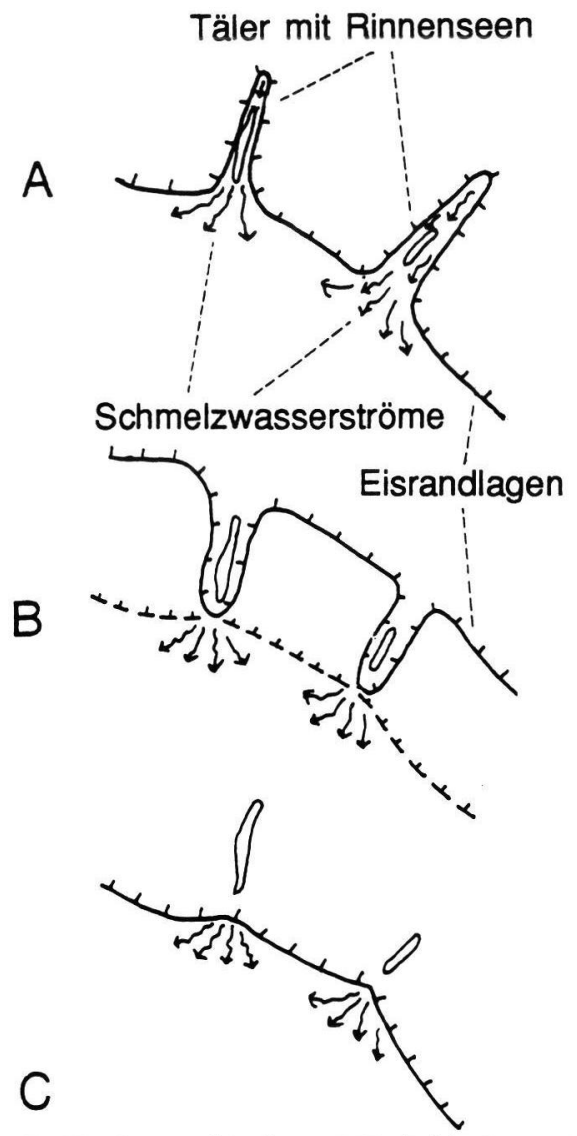

Abb. 3: Drei verschiedene Möglichkeiten, einen Eisrand zu konstruieren. Alle drei Zeichnungen sollen dieselbe Landschaft darstellen; die gestrichelte Randlage in (B) markiert eine frühere Eisrandlage, die der Randlage in (C) entspricht. (A) wird vielfach in Mecklenburg angewendet (FRANZ 1965, SCHUlz 1967, RüHBERG 1987), (B) entspricht GRIPPs Vorstellungen, wie sie in Schleswig-Holstein angewendet wurden, (C) ist die normale dänische Deutung. Nur (C) läßt sich mit den Gesetzen der Gletscherbewegung vereinbaren.

Fig. 3: Principles in common use when constructing ice margin lines, (A) in Mecklenburg, (B) in Schleswig-Holstein, and (C) in Denmark. The dot-anddash line in (B) represents "an earlier ice margin" (identical with the line in (C), so that two stages separated in, time are used to explain the same features which in (C) are ascribed to one and the same event. The mode used in (C) is the only one compatible with the physical (glaciological) laws.

geräumt worden ist, das in einem Tunnel unter dem Eis geflossen ist. Ussing hat sich diesbezüglich nicht klar ausgedrückt, aber NORDMANN und GRY haben später in Vorträgen und Diskussionen hervorgehoben, daß der Strom sich auch geteilt oder verlagert haben könnte, wo- bei jeweils der ältere Lauf durch niederfallende Eisblöcke blockiert wurde. Das Zusammentreffen der Talenden mit den höchsten Punkten der Schwemmkegel genau an der Eisrandlinie findet sich an so vielen Stellen, daß es sich unmöglich um einen Zufall handeln kann. Außer beim Tal westlich von Silkeborg, beim Hald-Sø-Tal und auch auf der Insel Sjælland (Abb. 6), sowohl bei kleinen als auch bei großen Tunneltälern.

2. Tunneltäler haben ein ungleichmäßiges Längsprofil. Das heißt, daß ihr Talweg auf und ab verläuft und wannenartige Vertiefungen enthält, die heute von Seen und Mooren erfüllt sind (Abb. 14). Daher können diese Täler nicht durch einen normalen Fluß erzeugt worden sein. In einem Rohr (Tunnel) kann dagegen im Unterschied zu einem subaerischen Wasserlauf das Wasser lokal aufwärts fließen. Das unausgeglichene Längsprofil belegt allerdings nur, wie ein Tunneltal nicht entstanden ist. Auch Grabenbrüche weisen ein unausgeglichenes Längsprofil mit eingeschalteten Seen auf.

3. Täler nach Art von Ussings Tunneltälern findet man nur in Nord- und Ostjütland, nicht im Südwestteil der Halbinsel. Ihre Verbreitung ist auf den Teil Jütlands beschränkt, der in der letzten Eiszeit vom Eis bedeckt worden ist. Die größeren dieser Täler enden an der mitteljütischen Eisrandlinie; keines von ihnen läßt sich weiter nach Südwesten verfolgen, auch nicht in begrabenem Zustand. Alle Erosionstäler, die sich südwestlich der Eisrandlage befinden, haben die Form normaler Flußtäler mit ebenem Talboden und kontinuierlichem Gefälle des Talwegs.

4. Am Boden der Tunneltäler oder in deren Verlängerung finden sich oft Oser und andere Rücken aus Schmelzwasserablagerungen (siehe Abb. 6 und 15). Oser stellen gewissermaßen Abgüsse des letzten lokal ausgebildeten Tunnels im Eis dar. Da die Oser schmaler sind als die Tunneltäler, drängt sich der Gedanke auf, daß die Schmelzwässer nicht das gesamte Tunneltal ausgefüllt haben. Dies stimmt mit den Erkenntnissen der heutigen Glaziologie überein.

5. Kein Tunneltal ist länger als $70 \mathrm{~km}$. Die meisten sind sogar deutlich kürzer. Das paßt zu der Tatsache, daß der Schmelzwasserabfluß 

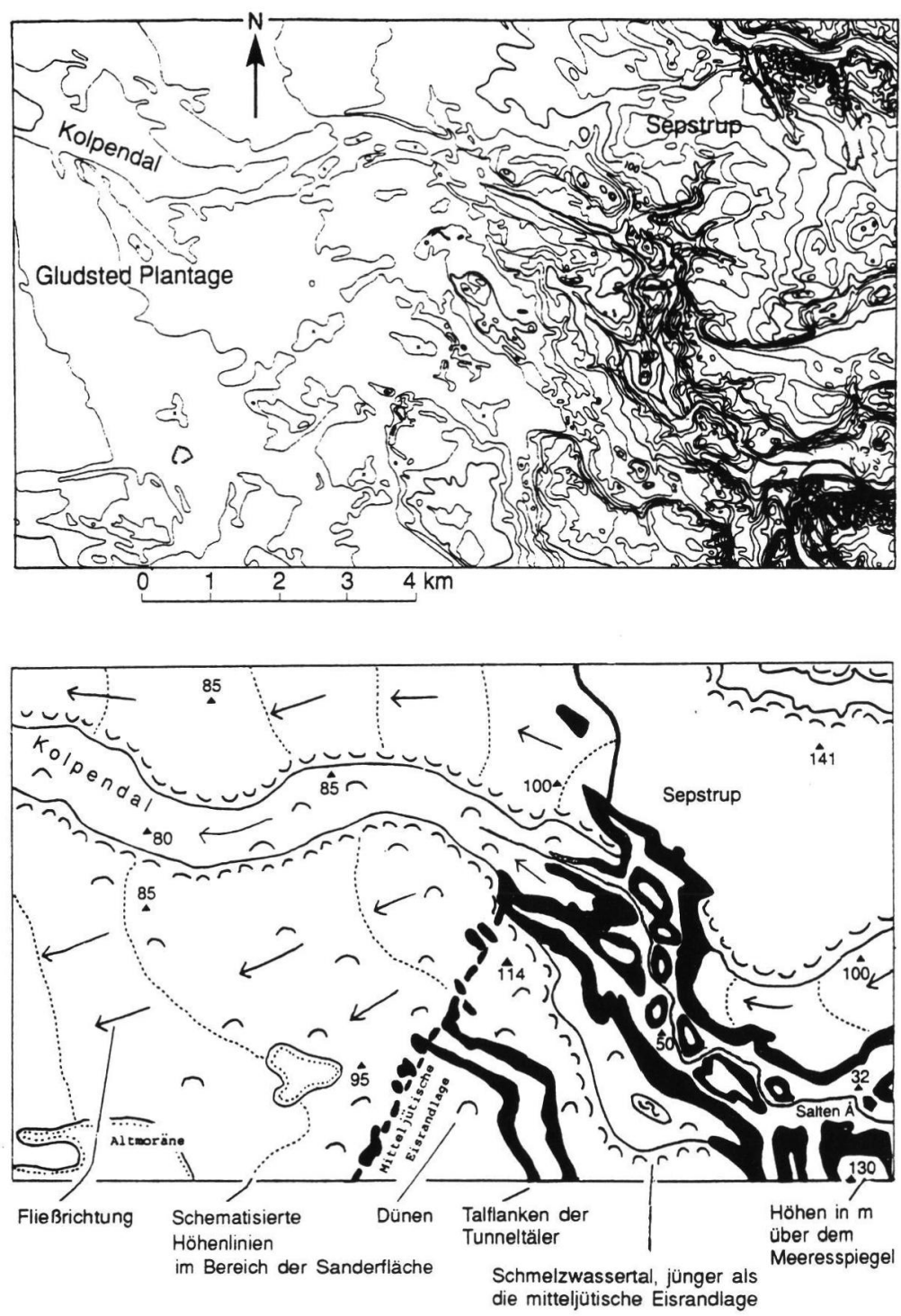

Abb. 4: Isohypsendarstellung (oben) und geomorphologische Skizze (unten) des Überganges zwischen dem Saltenå-Tunneltal und der westlich vorgelagerten Sanderfläche, südwestlich von Silkeborg (Jütland). Vervielfältigt mit Genehmigung Nr. A 246-96 von Kort-og Matrikelstyrelsen (Zeichnung: Der Verfasser).

Fig. 4: Contour map (above) and geomorphological sketch (below) of the transition between the Saltenå tunnel valley and the sandur plain west of the end point of this valley, SW of Silkeborg, Jutland. On the sketch below, the tunnel valley slopes are marked by a dark screen; a row of black dots represents the outermost Weichselian ice margin; dotted contour lines mark the sandur plain; Kolpendal is a younger erosion valley cut down into the sandur; "Altmoräne" is low hilly landscape formed during the Saalian glaciation.

unter dem Eis auf eine mehrere Zehner von Kilometern breite Zone am Rand des Inlandeises begrenzt ist (= die Ablationszone).
Keiner von Ussings fünf Punkten konnte ernsthaft entkräftet werden; dennoch gab es eine Reihe von Einwänden.

A) Das Gletschereis wurde als weitgehend wasserdicht aufgefaßt. Im Sommer entsteht an der Gletscheroberfläche Schmelzwasser. Es wurde behauptet, daß dieses Wasser nur durch Spalten in das Eis einsickern könne. Da Spalten nicht tiefer als 30 bis $40 \mathrm{~m}$ reichen (größere Tiefen werden durch den Eisdruck verhindert), wurde gefolgert, daß das Wasser die Gletschersohle nicht erreichen könne. Trotzdem wurde zum Teil behauptet, daß Tunneltäler und Oser entstehen, wo das Wasser in Spalten an der Gletschersohle abfließt. Spalten, die über eine Entfernung von $10-30 \mathrm{~km}$ von der Gletscheroberfläche bis zur Gletschersohle reichen, gehören jedoch in den Bereich der Phantasie; mit der aus Island bekannten Neigung der Gletscheroberfläche können $40 \mathrm{~m}$ tiefe Spalten die Gletschersohle nur im äußersten, etwa 0,5 $\mathrm{km}$ breiten Randbereich des Eisschildes erreichen. Das Mißverständnis liegt darin, daß die Masse des Wassers nicht durch Spalten zur Gletschersohle gelangt, sondern auf andere Weise (siehe unten).

B) Tunnel unter dem Eis können höchstens $30-50 \mathrm{~m}$ breit sein, sonst würden sie zusammenbrechen. Ein $1 \mathrm{~km}$ breiter Tunnel (z.B. im HaldSø-Tal) wurde als physikalisch unmöglich angesehen. Dabei wurde übersehen, daß es sich nicht um einen echten "Hohlraum" handelt. Das Wasser ist spezifisch schwerer als Eis und kann daher das Eis anheben, ohne daß dabei 


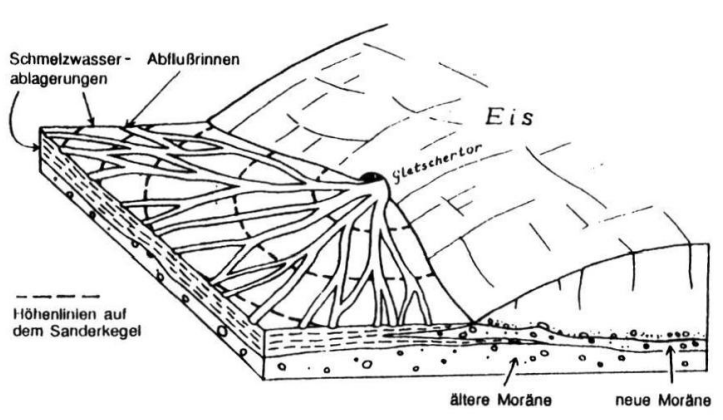

Abb. 5: Entstehung eines Sanderkegels. Der Sander wird aus dem Material aufgeschüttet, das das Schmelzwasser mitführt. Das Gefälle des Kegels ist vom Gletschertor fort gerichtet.

Fig. 5 :Genesis of a sandur cone. Contour lines show that the cone surface is inclined away from an apex where the mouth of the subglacial stream is found ("Gletschertor"). Neue Moräne = recently deposited till. Ältere Moräne $=$ older till .

große (hohe, breite) Hohlräume unter dem Eis entstehen - die sogenannten Hohlräume sind wassererfüllt. 1996 haben wir einen Beweis dafür bekommen, daß solche wassergefüllten Hohlräume unter dem Eis existieren können: Nach dem Vulkanausbruch bei Bardarbunga (Vatnajökull, Island) dauerte es einen Monat, bis die dadurch entstandenen ungeheuren Wassermengen (mehrere $\mathrm{km}^{3}$ ) durch Gletschertore über den Skeidarasandur abflossen. In der Zwischenzeit muß das Wasser in Hohlräumen unter dem Eis aufgestaut worden sein.

C) Die Sohle des Hald Sø liegt $23 \mathrm{~m}$ unter dem Meeresspiegel. Der höchste Punkt des proglazialen Sanderkegels bei Skelhøje, nur $2 \mathrm{~km}$ vom See entfernt (Abb. 2), liegt dagegen $80 \mathrm{~m}$ über dem Meeresspiegel. Wenn Ussings Idee stimmen soll, muß das Schmelzwasser hier auf eine Entfernung von $2 \mathrm{~km}$ einen Anstieg von $103 \mathrm{~m}$ bewältigt haben, das heißt, eine Steigung von 1:20. Das sah man - ohne eigentlich darüber zu diskutieren - als unmöglich an. Auch diese Vorstellung ist irreführend; darauf soll im folgenden eingegangen werden.

1972 berechnete SHREvE den Eisdruck und den Wasserdruck und kombinierte die Ergebnisse mit vorliegenden Beobachtungen. Eines der Ergebnisse von SHREve widerspricht direkt dem Argument (A) der Geologen: Es ist nicht richtig, daß das Gletschereis wasserdicht ist. Die Eisbewegung bewirkt, daß kleine Zwischenräume zwischen den Eiskristallen entstehen. Das Schmelzwasser sickert durch diese feinen Hohlräume nach unten. Die kleinen Hohlräume vereinigen sich zu größeren. Die Fließgeschwindigkeit, und damit die transportierte Wärmemenge, und damit das Schmelzen der Eiswände, sind in engeren Hohlräumen geringer als in größeren. Das Ergebnis ist ein baumartig verzweigtes Entwässerungssystem, dessen Hauptäste durch die Aufnahme des Wassers aus den schmalen Seitenästen allmählich nach unten breiter werden (Abb. 10). Diese Röhren haben nichts mit Spalten zu tun. Der Eisdruck kann sie nicht schließen, solange sie wassererfüllt sind - unabhängig davon, wie tief im Eis sie verlaufen. Der Wasserdruck ist nämlich überall größer als der Eisdruck, weil das Wasser spezifisch schwerer als Eis ist.

In "temperierten“ Gletschern hat die Gletscherbasis das ganze Jahr über eine Temperatur nahe $0^{\circ} \mathrm{C}$. Während die Lufttemperatur im Jahresgang kräftigen Schwankungen unterliegen kann (ein Beispiel aus Jotunheimen, Norwegen: mittlere Januartemperatur $-14^{\circ} \mathrm{C}$; mittlere Julitemperatur $+5^{\circ} \mathrm{C}$ ), pflanzen sich die Schwankungen im Inneren des Eises nur langsam fort und werden mit zunehmender Tiefe immer geringer. In $25 \mathrm{~m}$ Tiefe ist ein völliger Ausgleich erreicht. Hier entspricht die Temperatur das ganze Jahr über dem Jahresmittelwert der Temperatur (im genannten Beispiel: $-5^{\circ} \mathrm{C}$ ). Unterhalb dieser Tiefe bewirkt die Zufuhr von Erdwärme und die Reibungswärme vom Gleiten des Gletschers über den Untergrund, daß die Temperatur des Eises nach unten hin ansteigt. Alles deutet darauf hin, daß Schmelzwasserröhren, falls sie in temperierten Gletschern die "kalte Schicht" in 25 m Tiefe überwinden können, auch bis zur Gletschersohle gelangen können.

Im Winter kommt das Versickern von Wasser von der Gletscheroberfläche zum Stillstand. Die schmalen Hohlräume frieren zu oder werden durch die Gletscherbewegung geschlossen. Im nächsten Sommer öffnen sich allmählich neue Röhren. Gletschermühlen entstehen dort, wo Bäche an der Gletscheroberfläche auf eine Spalte treffen. Die Gletscherspalten und -mühlen sind aber nicht gleichmäßig an der Gletscheroberfläche verteilt. Von großen Teilen der Gletscheroberfläche kann das Schmelzwasser keine Gletschermühle erreichen. Bäche an der Gletscheroberfläche und Gletschermühlen sieht man vor allem im Frühjahr. Im Sommer verringert sich ih- 


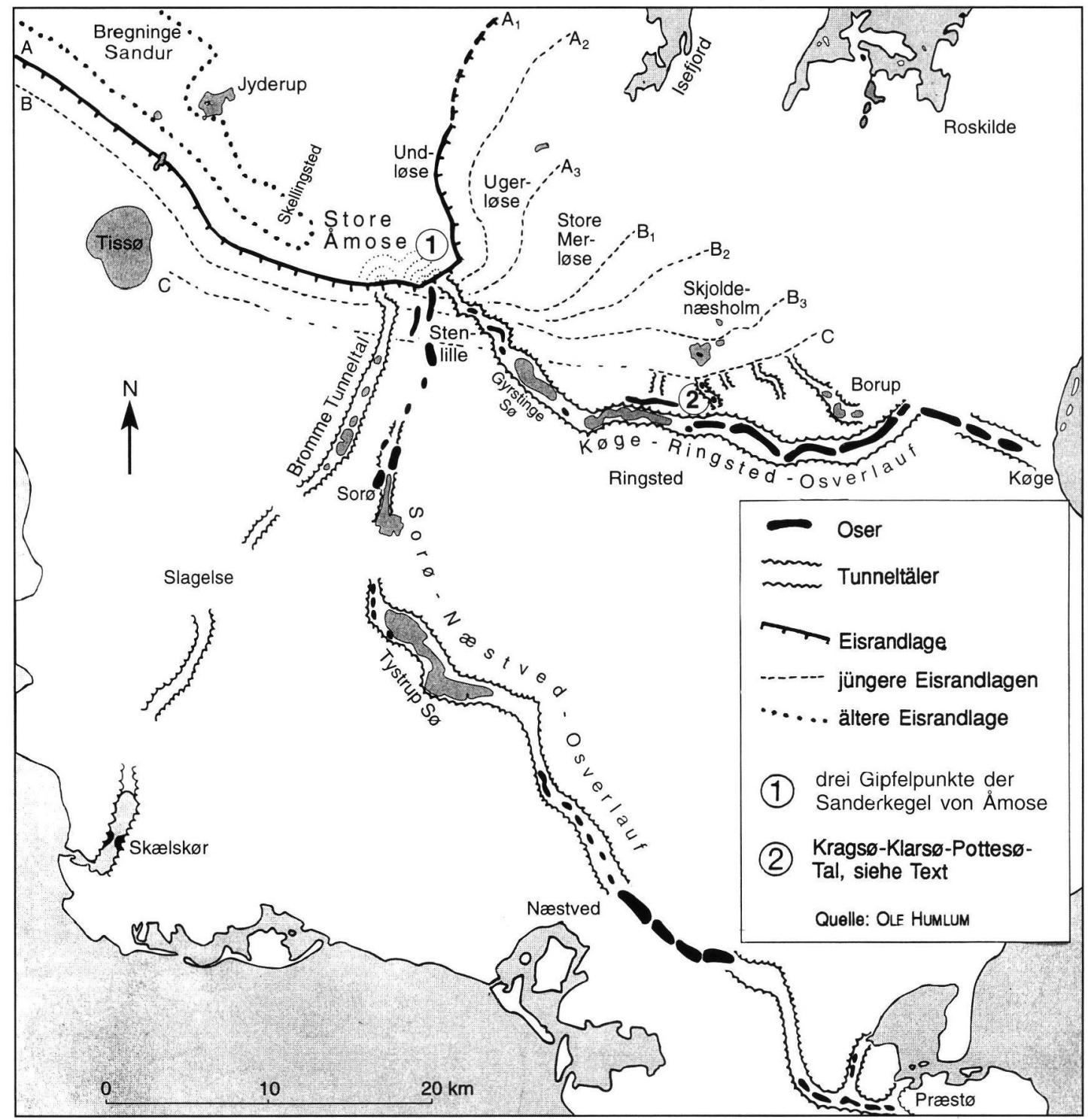

Abb. 6: Drei große subglaziale Wasserläufe mündeten gegen Ende der letzten Eiszeit südlich von Åmosen, Sjælland. Für jedes der drei Tunneltäler läßt sich auf der vorgelagerten Sanderfläche ein höchster Punkt mit groben Kiesablagerungen am ehemaligen Eisrand nachweisen. Bei Køge - Ringsted und Sorø - Næstved finden sich Dänemarks beste Beispiele für die Kombination von Osern und Tunneltälern. Im Bromme-Tunneltal finden sich keine Oser; es muß aber gleichzeitig mit den beiden anderen Tunneltälern gebildet worden sein (Zeichnung: Der Verfasser).

Fig. 6: On the island Sjælland three large subglacial streams were simultaneously active during the "Baelthav" ice advance late in the Weichselian glacial. The mouths of the streams were situated close to one another, $\mathrm{S}$ of the Store Amose bog. Three sandur cone apexes are found here, designated by their content of coarse meltwater gravel (1). The toothed line: main ice marginal zone in the area; dotted line: an older ice margin zone; dashed lines: slightly younger ice margin zones. Along the lines Sorø-Næstved and Køge-Ringsted, the subglacial streams can be traced as alternating stretches of eskers (black) and "incisions" (tunnel valleys) (toothed lines), or as eskers situated at the bottom of such valleys. The Bromme tunnel valley contains no esker. (2): the Kragsø-Klarsø-Pottesø valley, see text p. 16. The logically coherent genesis of all these features was shown by Ole Humlum 1976. The Sorø-Næstved and Køge-Ringsted subglacial stream tracks are the best Danish examples of a combined occurrence of eskers and tunnel valleys. See also fig. 15. 
re Wasserführung, da das Schmelzwasser sich andere Wege sucht: die oben erwähnten kleinen Hohlräume öffnen sich wieder.

Alle Hohlräume unter dem Eis bilden gemeinsam ein System kommunizierender Röhren. Die Gesetzmäßigkeiten, die für kommunizierende Röhren gelten, sollen hier kurz aufgeführt werden:

Im einfachsten Fall (Abb. 7) haben wir es nur mit einem einzelnen Gefäß zu tun. Das wäre ein Modell der Verhältnisse im Meer oder in einem See. Der Wasserdruck (der hydrostatische Druck) ist um so größer, je tiefer im Wasser der Punkt liegt. Die potentielle Energie ist dagegen im oberen Teil des Gefäßes hoch, im unteren niedrig. Die Summe von Druck und potentieller Energie ist

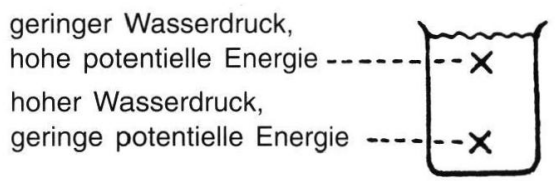

Abb. 7: Hydrostatischer Druck und potentielle Energie in einem Gefäß.

Fig. 7: Hydrostatic pressure and potential energy in a water tank.

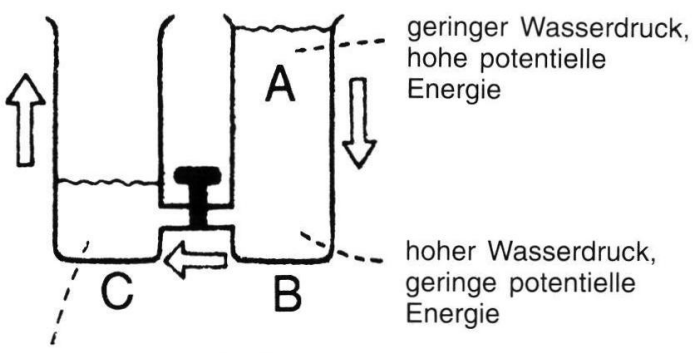

geringer Wasserdruck, geringe potentielle Energie

Abb. 8: Druck, und potentielle Energie in zwei Gefäßen, die mit einem Hahn verbunden sind. Pfeile: Strömung, wenn der Hahn geöffnet wird.

Fig. 8: Pressure and potential energy in two water tanks connected through a tap. Arrows: Stream when the tap has been opened.

überall in dem Gefäß gleich. Dieses Gleichgewicht sorgt dafür, daß sich keine Strömung ausbildet.

Die nächste Skizze (Abb. 8) zeigt zwei Behälter. In dem einen steht das Wasser höher als in dem anderen. Die Verbindung zwischen den beiden Behältern ist mit einem Hahn versehen. Solange der Hahn geschlossen ist, herrschen Verhältnisse wie auf Skizze 1, und es gibt keine Strömung. Wenn der Hahn geöffnet wird, schaffen wir ein Gefälle in der Summe des Drucks und der potentiellen Energie vom Boden des rechten Behälters zum Boden des linken Behälters. Eine Strömung von (B) nach (C) setzt ein. Der Wasserdruck bei (B) sinkt; das höhergelegene Wasser bei (A) kann nun nach unten sinken. Im linken Behälter steigt die Wassersäule, bis die beiden Wasserspiegel auf gleicher Höhe sind.

Die Röhren in einem Gletscher sind kein geschlossenes System, weil Wasser aus dem Gletschertor fließt und im Sommer durch Schmelzen an der Gletscheroberfläche neues Wasser durch Versickerung (in das Eis hinein) hinzugefügt wird. Den-

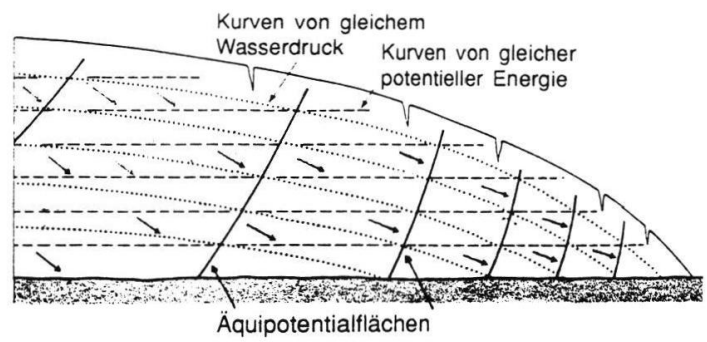

Abb. 9: Konstruktion von Äquipotentialflächen in einem Gletscher (nach JoHANNES KRÜGER 1989).

Fig. 9: Construction of equipotential planes through a glacier. Horizontal dashed lines: planes of equal potential energy. Inclined dotted lines: planes of equal water pressure. Full-drawn lines: equipotential planes. After Johannes KrÜger 1989).

noch lassen sich die Gesetze, die für kommunizierende Röhren gelten, mit guter Annäherung auch auf dieses System übertragen.

Die Summe von Wasserdruck und potentieller Energie wird Potential genannt und mit dem griechischen Buchstaben $\Phi$ (phi) bezeichnet. Das Wasser fließt von Gebieten mit hohem zu Gebieten mit geringem $\Phi$. Den Verlauf der Äquipotentialflächen in einem Gletscher zeigt Abb. 9. Das Wasser fließt rechtwinklig zu den Potentialflächen, so wie es durch die Pfeile in Abb. 10 angedeutet wird.

Von dem Punkt, wo das Wasser die Gletschersohle erreicht, strömt es in Richtung Gletschertor. Abb. 10 zeigt, daß das auch der Fall ist, wenn die Gletschersohle in Richtung Gletschertor ansteigt. Die maximale Steigung, die das Wasser überwinden kann, wird durch die Neigung der Äquipotentialflächen bestimmt. Wenn die äußeren Umstände es dazu zwingen, kann das Wasser schräg zu den Äquipotentialflächen fließen, aber nie entgegen den $\Phi$-Flächen. 


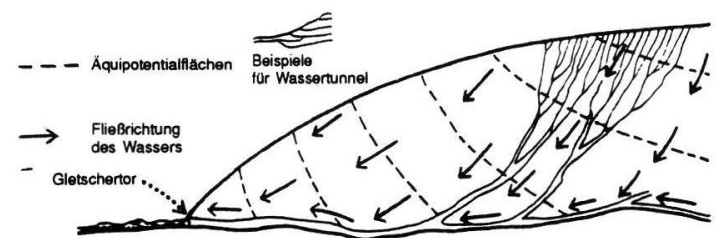

Abb. 10: Äquipotentialflächen und Wasserbewegung in einem Gletscher nach dem Modell von SHREve (1972).

Fig. 10: Equipotential planes and water movement through a glacier according to the model of SHREvE (1972). Dashed lines: equipotential planes. Arrows: stream directions. Examples of water pipes (tunnels) are shown.

Aus der Abb. 9 kann man berechnen, daß die Neigung der $\boldsymbol{\Phi}$-Flächen (in Richtung auf die Mitte des Eisschildes) etwa $11 \mathrm{mal}$ so groß ist wie die Neigung der Eisoberfläche an dieser Stelle. Damit läßt sich der Streitfall um den dänischen Hald $S \emptyset$ lösen. Die Äquipotentialfläche, die sich seinerzeit über dem See befunden hat, muß, wenn das Wasser auf eine Entfernung von $2 \mathrm{~km}$ um $103 \mathrm{~m}$ aufsteigen sollte, eine Neigung von mehr als 1:20 gehabt haben. Das bedeutet, daß die Eisoberfläche an dieser Stelle eine Neigung von mehr als 1:220 aufgewiesen haben muß. Die äußersten $2 \mathrm{~km}$ der

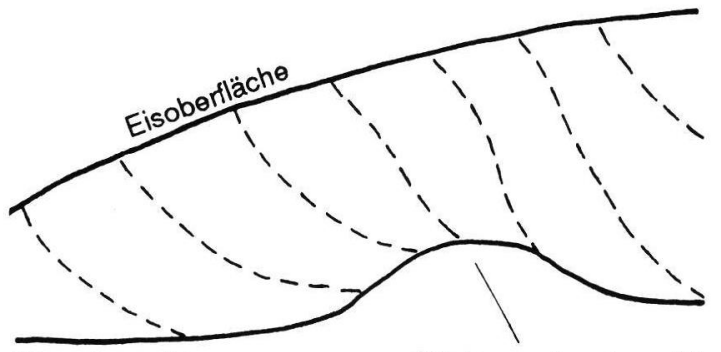

Gletschersohle

Rücken unter dem Eis

Abb. 11: Verlauf der $\Phi$-Flächen, wenn das Eis ein Hindernis passiert.

Fig. 11: Course of the equipotential planes where the glacier passes an obstacle.

heutigen Gletscher Islands haben aber eine durchschnittliche Oberflächenneigung von 1:10.

Ihr Gefälle ist also zweiundzwanzigmal größer als erforderlich, um eine Hohlform wie den Hald Sø entstehen zu lassen.

Sowohl der Eisdruck als auch der Wasserdruck nimmt auf der Luvseite eines Buckels unter dem Eis zu. Die Abb. 11 zeigt, wie die $\Phi$-Flächen an dieser Stelle vorwärts verbogen werden, und dann auf der Leeseite stärker als durchschnittlich zurückbiegen. Abb. 12 zeigt dieselbe Situation wie Abb. 11, aber von oben gesehen. Die gestrichelten Linien in diesem Bild sind nicht die $\Phi$ Flächen, sondern deren Schnittlinien mit der Gletschersohle. Man sieht, daß sich die subglazialen Schmelzwasserströme in den Niederungen sammeln. Dennoch können kleine Ströme einen Höhenrücken kreuzen. Sowohl Abb. 11 als auch Abb. 12 zeigen, daß das Gefälle von $\Phi$ über Höhenrücken am größten und über Vertiefungen am geringsten ist. Die Strömungsgeschwindigkeit erhöht sich deshalb im Abstrom eines Höhenrückens und verringert sich in Niederungen. Ein Os ist daher in Niederungen groß und sandig, auf Höhenrücken dagegen klein und kiesig ausgebildet, oder es fehlt sogar völlig. Deutliche Beispiele für diesen Zusammenhang findet man z. B. auf Fyn (Dänemark).

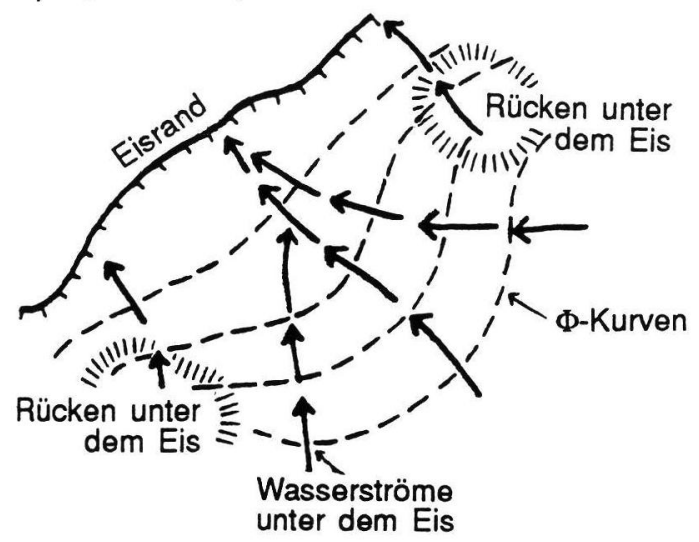

Abb. 12: Verlauf der $\Phi$-Flächen auf der Gletschersohle, wenn Hindernisse (Rücken) unter dem Eis auftreten.

Fig. 12: Course of the equipotential lines at the bottom of a glacier (i. e., the intersection lines between the equipotential planes and the glacier base) where obstacles (hills) on the glacier bed are found. Explanation of signs see fig. 13.

Wo der Eisrand Loben bildet, ist das Eis in der Mitte der Loben jeweils dicker als in den zurückspringenden „Kerben“ des Eisrandes. Oft sind Höhenrücken unter dem Eis durch ihre Bremswirkung auf die Eisbewegung die Ursache dafür, daß sich der Eisrand in Loben (Zungen) aufgliedert. Der Zusammenhang zwischen diesen Verhältnissen ist in der Abb. 13 verdeutlicht. Man sieht, daß die $\Phi$-Kurven auf großen Strecken parallel zum Eisrand verlaufen, daß aber Höhenrücken unter dem Eis Abweichungen von diesem Prinzip bewirken. Die Eisoberfläche steigt in den Kerben steiler an als in den Loben; die $\Phi$-Flächen liegen daher im Bereich der Kerben am dichte- 
sten zusammen. Hier fließt das Wasser am schnellsten; infolgedessen kommt es hier zu verstärkter Erosion. An dieser Stelle entstehen die größten Tunneltäler. Die meisten Tunnel unter

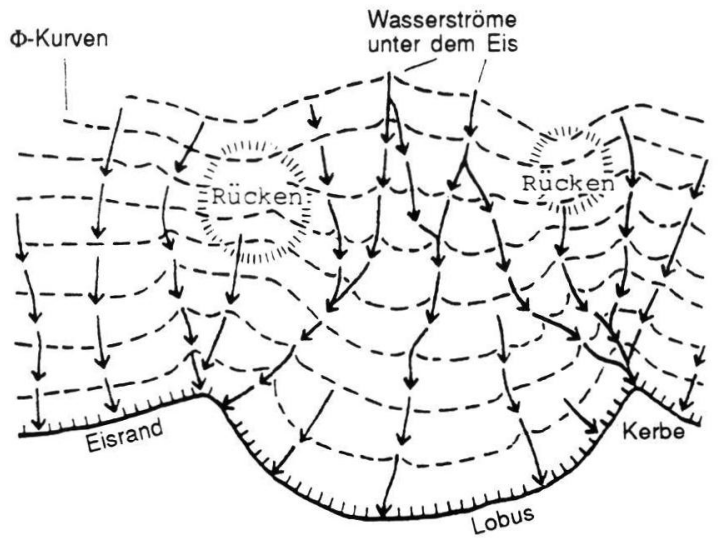

Abb. 13: Zusammenhang zwischen Eisrand und $\Phi$-Kurven-Verlauf, wenn der Eisrand in Loben aufgeteilt ist.

Fig. 13: Courses of the equipotential lines (see fig. 12) and the subglacial stream pattern where the ice front is divided into lobes. Eisrand $=$ ice margin. Kerbe $=$ inward-bent angle. Rücken $=$ obstacle (hill). Dashed lines $=$ equipotential lines at the glacier bottow. Arrows = subglacial streams .

dem Eis haben ihre Mündung in oder nahe diesen Kerben. Hier befinden sich daher auch die größten Gletschertore.

Ein Zusammenhang zwischen Eistunneln (Osern), Eisrandkerben, Gletschertoren und Sanderflächen wurde bereits um das Jahr 1900 nachgewiesen, als die eiszeitlichen Landschaften Dänemarks, Mecklenburgs und Pommerns zum ersten Mal kartiert wurden (siehe auch Abb. 15). Tiefere Erkenntnisse über die Ursachen der Formung mußten warten, bis weitere Fortschritte in der Glaziologie erzielt waren. Untersuchungen unter aktiven Gletschern lassen sich schwerer durchführen als eine Kartierung der heimatlichen Landschaft. Das wichtigste an SHREves (1972) Feststellungen ist, daß seine Skizzen (siehe Abb. 13) genau auf das bekannte Landschaftsbild in Dänemark und Norddeutschland übertragbar sind. Sie illustrieren die physikalischen Prozesse, die dem Entstehen dieser Landschaft zugrunde liegen. Ein wichtiger Punkt ist, daß sowohl Tunneltäler als auch Oser dem Verteilungsmuster folgen, das SHreve aufgezeigt hat (siehe z.B. Abb. 6), und daß das Muster sowohl für breite als auch schmale Tunneltäler gilt. Die großen Tunneltäler haben denselben morphologischen Charakter, z. B. denselben Typ von Talsohle und dasselbe Verhältnis zur umgebenden Landschaft wie die kleinen. Es beinhaltet meiner Meinung nach einen logischen Denkfehler, wenn man versucht, große und kleine Tunneltäler auf unterschiedliche Weise zu erklären, wie das einige Verfasser bis in jüngste Zeit getan haben (Krüger 1989, LARSEN, KrONBORG \& Bender 1979, Larsen \& Kronborg 1994).

In Ländern, in denen der Untergrund aus Festgestein besteht, sind Tunneltäler nur selten anzutreffen. Parallelen zu unseren Taltypen findet man dagegen in früher vergletscherten Gebieten mit einem Untergrund aus Lockergestein. Hierzu zählen z. B. die nördlichen USA, das südliche Kanada, das westliche Rußland und große Teile Polens. Sugden \& John (1979) beschreiben englische Beispiele und heben hervor, daß die subglaziale Schmelzwassererosion wahrscheinlich viele Spuren hinterlassen hat; die Schwierigkeiten liegen darin, dies zu beweisen.

Schon um 1900 kannte man tief eingeschnittene, begrabene Täler aus der eiszeitlichen Landschaft Norddeutschlands. Von 1960 bis 1980 wurden so viele Bohrungen abgeteuft, daß man sowohl Längs- als auch Querprofile durch diese Täler konstruieren konnte (z. B. Kuster \& MEYer, 1979). Vor allem Ehlers (1994) hat die Beobachtungen zusammengefaßt und die Entstehung dieser Formen rekonstruiert. So viel kann gesagt werden:

1. Die Täler sind viele $\mathrm{km}$ lang, von 30 bis über $400 \mathrm{~m}$ tief und haben ein unausgeglichenes Längsprofil. Deshalb können sie nicht als normale Flußtäler entstanden sein.

2. Die Täler bilden ein unregelmäßiges Netzwerk. An verschiedenen Stellen kreuzen sie sich. Andere Täler enden plötzlich blind, obwohl sie wenige Kilometer vor ihrem Endpunkt noch breit und tief sind. LYKKE-ANDERSENS Idee, daß es sich um durch Gletschererosion übertiefte Flußtäler handelt (LyKKE-ANDERSEN, 1986), ist deshalb nach EHLERs (1994) zurückzuweisen. Das Talsystem weist keine Ähnlichkeit mit einem Flußsystem auf.

3. Die Täler der beschriebenen Art liegen alle innerhalb des ehemaligen Vereisungsgebietes in Norddeutschland, keines außerhalb. Deshalb muß man davon ausgehen, daß das Eis etwas mit ihrer Entstehung zu tun hat.

4. Bei den Tälern kann es sich nicht um Grabenbrüche handeln. Die tieferen Schichten weisen keine entsprechenden Störungen auf. Die tertiären Schichten lassen sich unter den Tälern 
wie außerhalb in gleichbleibender Tiefe nachweisen.

5. Es sieht nicht so aus, als gäbe es einen engen Zusammenhang zwischen Tälern und Salzstrukturen. Einige Täler liegen zwischen den Salzstrukturen, andere queren sie.

6. Die Ablagerungen, mit denen die Täler verfüllt sind, stammen alle aus dem Quartär. Hauptsächlich handelt es sich dabei um Schmelzwassersande und Beckentone, zum Teil auch um Kies. Moränenähnliche Lagen (Diamiktone) sind selten und können in den meisten Fällen entweder als Abrutschmassen oder als Fließmoräne (Material, das in verflüssigtem Zustand aus der Umgebung in die Täler geflossen ist) gedeutet werden. In den oberen Teilen der Rinnenfüllungen kommen interglaziale Ablagerungen vor, sowohl in mariner als auch in limnisch-telmatischer Fazies.

7. Mit Hilfe der interglazialen Ablagerungen lassen sich die meisten der Täler drei Gruppen zuordnen: Die tiefsten und am steilsten eingeschnittenen stammen aus der Elster-Eiszeit, einige breitere und weniger tiefe aus der SaaleEiszeit. An Stellen, die in der letzten Eiszeit (Weichsel-Eiszeit) vom Eis bedeckt waren, kommen begrabene Täler vor, die außer durch eiszeitliche Ablagerungen mit postglazialen Schichten verfüllt worden sind. Daraus läßt sich zwanglos ableiten, daß diese in der Weichsel-Eiszeit entstanden sind. Im Gegensatz zu den älteren Rinnen sind die meisten Tunneltäler der Weichsel-Eiszeit jedoch heute noch im Gelände sichtbar.

Die unter 6. angeführten Punkte sprechen dagegen, daß Gletschererosion bei der Entstehung der Täler die entscheidende Rolle gespielt hat. Hinzu kommt, daß die älteren Schichten, die an den Talflanken ausstreichen, in der Regel scharf abgeschnitten sind (wie mit einem Messer) und nicht gestört sind. Aufschiebungen und andere Störungen, wie sie dem Eis zugeschrieben werden, kommen zwar vor, sowohl am Talboden als auch in den obersten Schichten an den Talflanken. Das Eis hatte demnach Auswirkungen auf die Täler und ihre Ausformung, aber die Störungen hatten einen untergeordneten Charakter.

Subglaziale Schmelzwassererosion bleibt offensichtlich als einzige Möglichkeit, um die Entstehung der begrabenen Täler zu erklären! Die Übereinstimmung mit den norddeutschen Rinnenseeund Fördentälern und mit den dänischen Tunneltälern ist so überzeugend, daß man kaum um eine gemeinsame Entstehung herumkommt. Einige der begrabenen Täler sind allerdings so breit und tief, daß kaum genügend Schmelzwasser auf einmal unter dem Eis existiert haben kann, um die Täler auszugraben. Deshalb vermuten EHLERs \& WingFelD (1991), daß es sich um plötzliche Ausbrüche großer Wassermengen gehandelt haben könnte, ähnlich den isländischen Gletscherläufen (jökulhlaups). Solange die Entstehung der Täler nicht feststeht, zieht EHLERs (1994) vor, sie als „Rinnen“ (incisions) zu bezeichnen anstatt als Tunneltäler.

Unter dem Einfluß der Erkenntnisse aus England (SHrEve 1972) und Deutschland ist inzwischen die Tunneltal-Theorie in Dänemark wieder neu belebt worden. Der wichtigste Vertreter dieser Erneuerung ist der Glaziologe Ole Humlum, Geografisk Institut, Kopenhagen, ohne dessen Hilfe dieser Artikel nicht hätte geschrieben werden können.

Das große Problem bei der Deutung der Tunneltäler, von GripP um 1920 bis EhlERs \& WingFielD 1991 und Larsen \& Kronborg 1994, war immer die große Breite der Täler. SHREve (1972) führt in einer Tabelle auf, wie groß die Wassermenge pro Minute sein muß, um einen Tunnel mit bestimmtem Durchmesser offen zu halten. Falls der Druck abnimmt, preßt das Eis die Tunnelwände nach innen. Um einen Tunnel von einem Durchmesser von $1 \mathrm{~km}$ offen zu halten, benötigt man eine Wassermenge etwa in der Größenordnung der Wasserführung der Donau in Rumänien. In Jütland finden sich aber Tunneltäler dieser Größe in einem Abstand von jeweils etwa $20 \mathrm{~km}$ auf der ganzen Strecke von Kolding über Viborg bis Bovbjerg (s. Abb. 1). Um die erforderliche Wassermenge bereitzustellen, müßten so viele Schmelzwasserströme von der Größenordnung der Donau gleichzeitig geflossen sein, daß, um sie zu speisen, das gesamte Inlandeis in Dänemark innerhalb von weniger als 10 Minuten geschmolzen wäre. Folglich muß man sich nach einer anderen Erklärung umsehen.

Die Vorstellung, daß die Täler nicht auf einen Schlag erzeugt worden sind, sondern sukzessive, und daß der Tunnel zu keinem Zeitpunkt die ganze Breite des Tales eingenommen hat, sondern schmaler gewesen ist und sich verlagert hat, wurde bereits mehrfach erwähnt (Abb. 15). Der Vorschlag ist alt, wurde aber bis vor kurzem nicht ernst genommen (KRÜGER 1989). Wenn wir mit EHLERS (1994) alle anderen Möglichkeiten ausschließen, bleibt vielleicht nur diese Lösung, obwohl erwogen werden muß, ob Gletscherläufe (jökulhlaups) bei der Bildung der Tunneltäler 
eine Rolle gespielt haben. Eine Betrachtung der Verhältnisse unter dem Inlandeis nach heutigem Wissensstand kann hier vielleicht das Verständnis der Vorgänge vertiefen:

Zunächst einmal wissen wir, daß die Schubspannung im Eis (der Eisdruck) einen Tunnel allmählich einengt, wenn der Wasserdruck darin nachläßt (oder wenn der Tunnel lufterfüllt wird). Es ist schwer zu beurteilen, wie rasch diese Einschnürung in Jütland während der Eiszeit abgelaufen ist, aber man kann nach Humlum (pers. Mitt.), wenn man die Bewegungsgeschwindigkeit der heutigen isländischen Gletscher in Betracht zieht, einen Wert von 5-15 m im Laufe eines Winters abschätzen.

Zum anderen wissen wir, daß die Unterschiede zwischen sommerlicher und winterlicher Wasserführung bei Gletscherflüssen viel größer sind als bei normalen Flüssen. Eine gewisse Menge Schmelzwasser wird das ganze Jahr über an der Sohle temperierter Gletscher produziert, während der Zufluß von der Gletscheroberfläche im Winter zum Erliegen kommt. Einer der Abflüsse des Hoffellsjökull auf Island wurde im Winter zu 4 m3/s gemessen. Im Sommer variierte die Wassermenge stark; das Maximum lag bei 150 m3/s, dem 38fachen des winterlichen Abflusses. Im Mai-Juni stieg die Wasserführung zunächst auf das zehnfache, bis schließlich das Haupt-Maximum um St. Johannis eintrat (KrÜGER 1989). Ein Tunnel an der Gletschersohle könnte wahrscheinlich nach sechs Monaten allmählicher Einschnürung eine solche Flut nur schwer fassen. Das Frühjahrsschmelzwasser ist sedimentreich, und da die Strömung sehr turbulent ist, hat das Wasser eine große Erosionskraft.

Die Lockersedimente Dänemarks und Norddeutschlands waren unter den Gletschern nicht gefroren; man muß sie sich als das ganze Jahr über wassergesättigt vorstellen. Es leuchtet ein, daß sich unter diesen Bedingungen ein neuer Tunnel parallel zum alten Abfluß schnell ausbilden könnte, und daß der neue Tunnel sich zunächst in den Untergrund einschneidet anstatt sich einen Weg durch das Eis zu schmelzen. Im Laufe des Sommers würde sich der neue Tunnel vielleicht stabilisieren, wobei sich der alte Abflußweg eventuell schließen würde. Im folgenden Jahr könnte sich dieser Vorgang wiederholen. Die Strecke, entlang derer sich ständig neue Tunnel einschneiden, würde auf diese Weise jedes Jahr breiter. Blockierte Tunnel würden sich im Laufe weniger Jahre völlig schließen - nicht durch Wasser, das gefriert, und vielleicht auch nicht durch niederstürzende Eisblöcke, sondern durch das massive Gletschereis, das sich in den Hohlraum hineinpreßt. Wenn dieser Vorgang sich z.B. über 500 Jahre wiederholt, entstünde auf diese Weise eine breite Talzone, von der vielleicht $90 \%$ durch Gletschereis erfüllt wären.

Das Eis schmilzt hauptsächlich von oben. Die beinahe keilförmigen Eispartien, die von der Basis des Inlandeises in die Tunneltäler hineinragen, könnten als Toteis zurückbleiben und zuletzt im Verlauf des Abschmelzens durch jüngere Sedimente und vom Gletscher abgerutschtes Material begraben werden.

Auf diese Weise verstanden, bilden die Tunneltäler ein normales Glied der glazialen Landschaftsentstehung in Gebieten mit einem Untergrund aus unverfestigten Schichten. Die Entstehung der Tunneltäler kann dann als Ergebnis der extremen Schwankungen in der Schmelzwasserproduktion im Laufe des Jahres interpretiert werden, wie auch die Korngrößenschwankungen in unseren Kiesgruben und die Varven der Eisstauseen. Der beschriebene Prozeß setzt keine Grenzen dafür, wie breit oder tief ein Tunneltal werden kann (Abb. 15). Das Argument, daß die Täler zu breit seien, ist damit außer Kraft gesetzt. Der scheinbare Widerspruch dazwischen, daß die Täler aussehen, als seien sie von Wasser erodiert worden, und daß ihre charakteristischsten Abschnitte durch Toteis-Sackungen geprägt sind, ist aufgehoben.

Die Morphologie der dänischen Tunneltäler (s. Abb. 14) läßt sich durch den beschriebenen Prozeß zwanglos erklären: Längsrücken an der Talsohle unterteilen sie in schmalere Abschnitte; Seitenäste lassen sich in das Moränen-Hinterland verfolgen; neue Seitenarme kommen dazu; „Henkel" weichen zur Seite aus und schließen sich wieder an das Haupttal an. Abb. 14 zeigt all diese, und außerdem auch die oft beschriebenen Merkmale der Tunneltäler: Seen und Moore im Talverlauf und eine Wasserscheide quer zum Tal (zwischen Ring Sø und Sømose.) Siehe auch Abb. 15.

Für mich haben die übrigen Erklärungsversuche, die im Laufe der Zeit vorgeschlagen worden sind, den Charakter von Notlösungen: Sie können in Einzelfällen oder Grenzfällen Gültigkeit haben, aber es gibt Probleme, wenn man versucht, sie allgemein anzuwenden.

Die Vorstellung, daß Gletscherzungen die Täler ausgegraben haben, muß infolge der heutigen glaziologischen Erkenntnisse aufgegeben werden, und es muß hinzugefügt werden, daß sie 


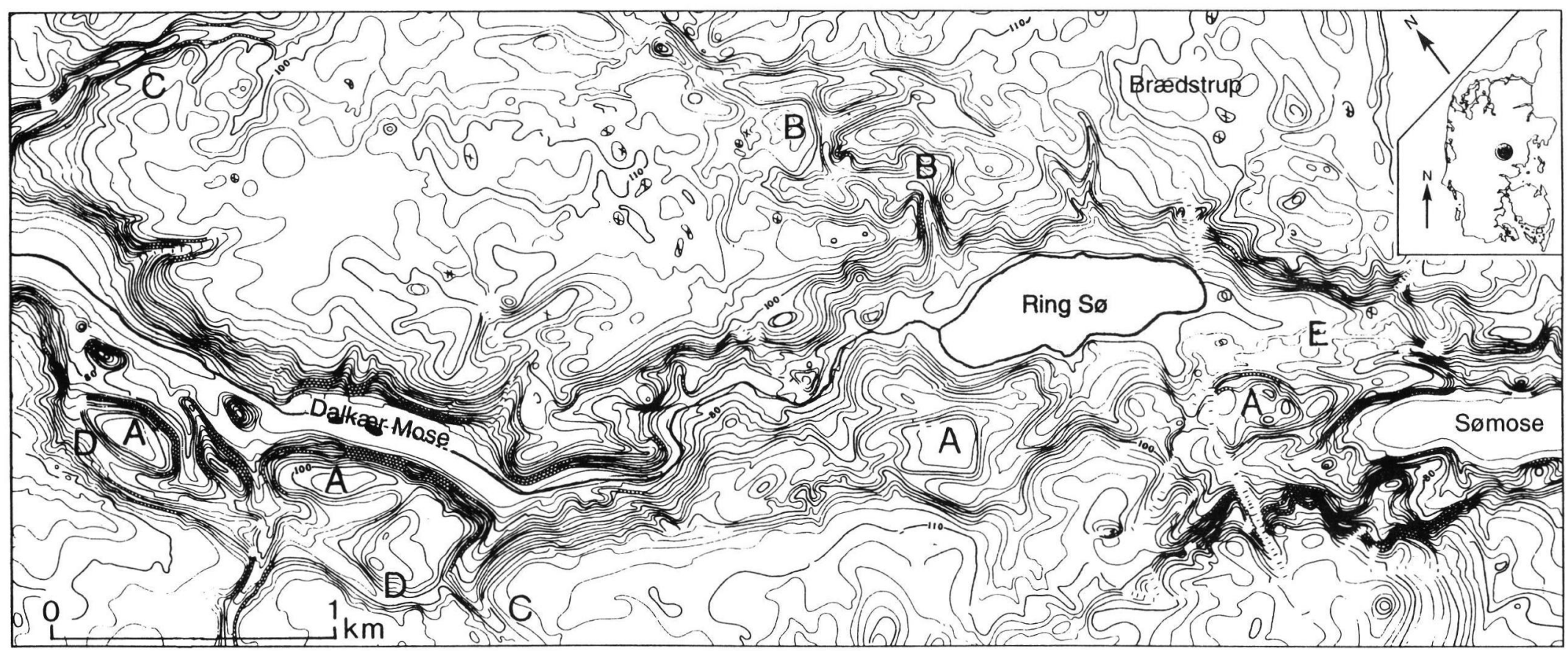

Abb. 14: Ein typisches Tunneltal in Jütland. Ausschnitt aus dem Isohypsenbild der Karte M 2710 Bræedstrup 1.20.000, verkleinert. Isohypsen im 2-mAbstand. Höhenangaben in Metern. Vervielfältigt mit Genehmigung Nr. A 246-96 von Kort- og Matrikelstyrelsen. (A) Rücken, die die Talsohle in mehrere Arme aufteilen; (B) Abzweigungen, durch die Wasser vom Haupttal aus in die Umgebung geströmt ist; (C) Seitenarme, durch die Wasser aus der Umgebung ins Haupttal geströmt ist; (D) henkelartige Nebenrinne; (E) heutige Talwasserscheide.

Fig. 14: A typical tunnel valley in Jutland. Section of the topographical map M 2710, reduced scale. Contour interval $2 \mathrm{~m}$. (A) Ridges subdividing the valley bottom into parallel tracks. (B) Valley tracks branching out into the surroundings. (C) Valley tracks (presumably) eroded by tributary streams running into the main course. (D) Handle-like lateral valley branches. (E) Present watershed. 


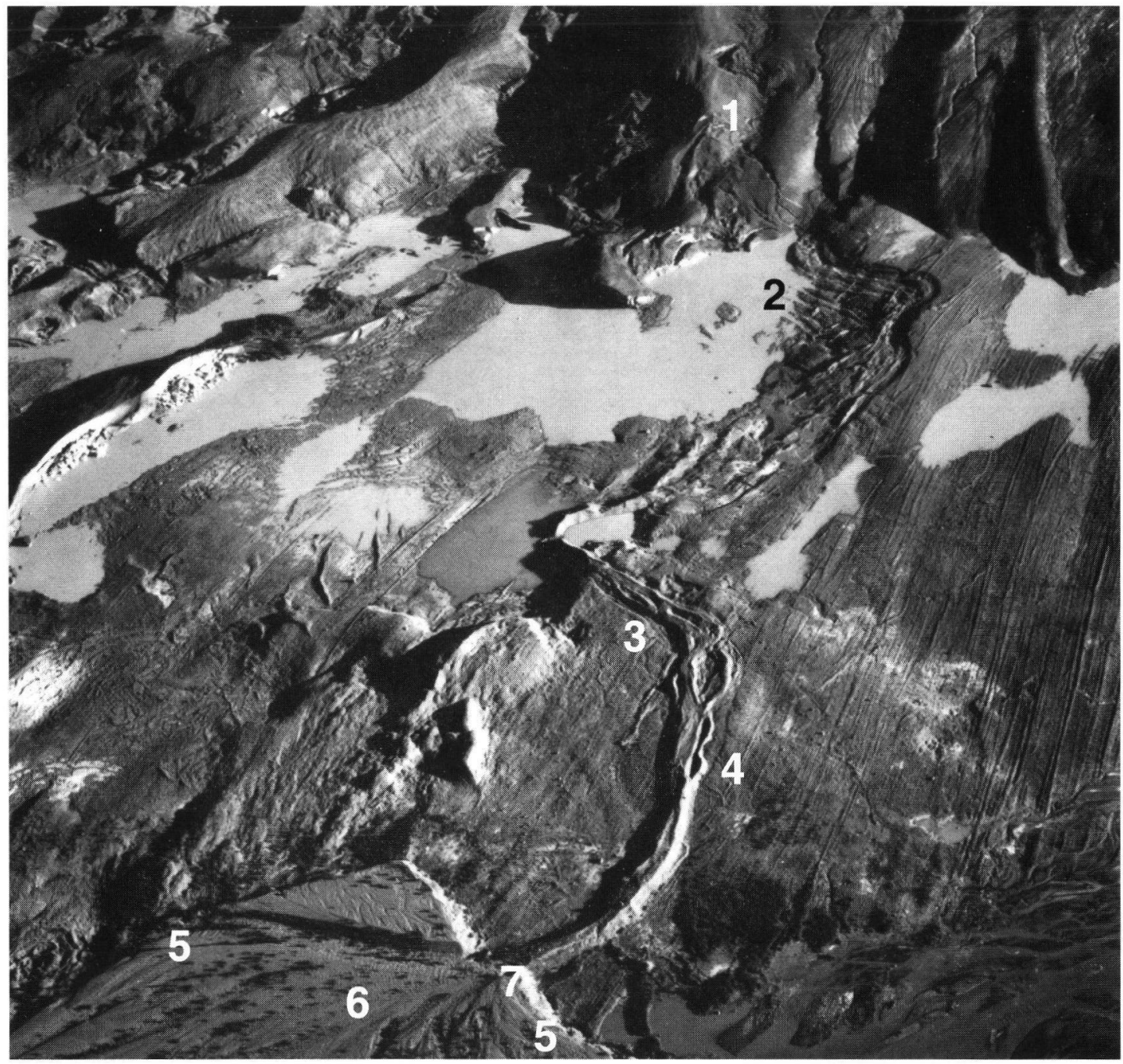

Abb. 15: Landschaft im Vorfeld des Woodworth Glacier im Tasnuna Valley, Alaska. Luftaufnahme vom 8. August 1938, 6.45 Uhr. 1 = Gletscher. 2 = durch subglaziale Schmelzwasserströme erodierte Rinnen (Tunneltäler). Beachten Sie bitte. daß die Rinnen von der tiefliegenden Wasserfläche links nach rechts auf höheres Gelände ansteigen. Das belegt, daß das Wasser bergauf gelaufen ist, als die Rinnen gebildet wurden. 3-4 = Os. Bei (3) sieht man, daß der Osrücken in einer breiten Rinne liegt, diese aber nicht ausfüllt. 5 = Position des Eisrandes, als das Os gebildet wurde. $6=$ Sanderfläche, die auf die von Ussing beschriebene Weise von einem Schmelzwasserstrom gebildet wurde, der sich vom höchsten Punkt am Gletschertor (7) aus in viele Flußarme aufgeteilt hat. Die dunklen Flecken sind Bäume. Quelle: BrADFORD WASHBURn, Museum of Science, Boston, USA.

Fig. 15: Landscape at the margin of the Woodworth glacier, Tasnuna valley, Alaska, 8. 8. 1938. (1) Glacier. (2) Valley tracks eroded by subglacial streams. Note that the tracks run from the water puddle at left upwards on to dry land at right, i.e. that the water did run uphill when the valleys were eroded. (3)-(4) Esker. By (3) it can be seen that the esker ridge lies in a broad furrow which it does not fill in, compare fig. 6. (5) Position of the ice margin when the esker was deposited. (6) Sandur plain, formed in the way shown on fig. 5. The dark dots are trees.

auch mit den allgemeinen Vorstellungen von der Wirkung des Eises im Widerspruch steht. Eiserosion kann im wesentlichen auf zwei Weisen wirken: a) dadurch, daß einzelne Partikel von der Gletschersohle aufgenommen werden; dies geschieht oft gleichzeitig über große Areale, denn die Temperaturverhältnisse an der Glet- 
schersohle bestimmen, ob das stattfindet oder nicht;

b) dadurch, daß Schollen von Untergrundmaterial an der Sohle des Gletschers festfrieren und vorwärts transportiert werden, ohne daß deren innere Struktur verändert wird. Die Schollen können eventuell zu bogenförmigen Höhenrücken (Endmoränen) aufgestapelt werden. Auch im zuletzt genannten Fall wirkt die Erosion über eine breite Fläche. Die Eintiefung schmaler, scharf begrenzter Rinnen ohne wesentliche Störung der angrenzenden Schichten liegt nicht in den Möglichkeiten des Eises. Dagegen wirkt die Wassererosion genau auf diese Weise - und deshalb sehen die Tunneltäler so aus.

LARSEN \& KRONBORG (1994) halten fest an der Vorstellung, die auch schon von LıRSEN, KRONBORG \& BENDER (1979) geäußert wurde, nämlich daß die Talbildung in Mitteljütland durch Bruchlinien im Untergrund (Verwerfungen) beeinflußt worden ist. Hierzu kann gesagt werden, daß der Untergrund in ganz Dänemark von Bruchlinien durchzogen ist. Die meisten verlaufen in nordwestsüdöstlicher Richtung, weil sie ein Teil der fennoskandischen Randzone (der sogenannten Sorgenfrei-Tornquist-Zone) sind (VARV 1992). Im Gebiet zwischen Horsens und Silkeborg verlaufen die Tunneltäler parallel zu dieser Bruchzone. Für die- ses Gebiet mag die Vorstellung von LARSEN \& KRONBORG zutreffen (siehe z.B. LYKKE-ANDERSEN, 1995). Aber auf der Strecke von Silkeborg über Viborg nach Lemvig drehen die Richtungen der Tunneltäler von Südost-Nordwest über Ost-West und Nordost-Südwest bis Nordnordost-Südsüdwest. Man sucht vergebens nach entsprechenden Erscheinungen im Verlauf der Bruchlinien. Die Übereinstimmungen in der Umgebung von Horsens sind ein Sonderfall, vielleicht ein Zufall. Dagegen verlaufen die Tunneltäler überall rechtwinklig zu Ussings Eisrandlinie, nicht nur generell, sondern auch wenn man deren örtliche Bögen mit in Betracht zieht.

LARSEN \& KRONBORG (1994) führen an, daß die jütischen Tunneltäler rechtwinklig zu den Formationsgrenzen im Untergrund verlaufen. Dazu ist zu sagen, daß es auch typische Tunneltäler auf Sjælland und Lolland gibt, wo dies nicht zutrifft. Die Tunneltäler verlaufen dagegen überall in der Bewegungsrichtung des Eises, unter dem sie vermutlich entstanden sind. Ussings Eisrandlage verläuft überwiegend parallel zu den Formationsgrenzen, aber das kann daran liegen, daß die beiden Phänomene eine gemeinsame Ursache haben: Sowohl die Schichtgrenzen als auch die Eisrandlinie halten - im großen und ganzen - einen konstanten Abstand vom Festgestein der skandinavischen Halbinsel.

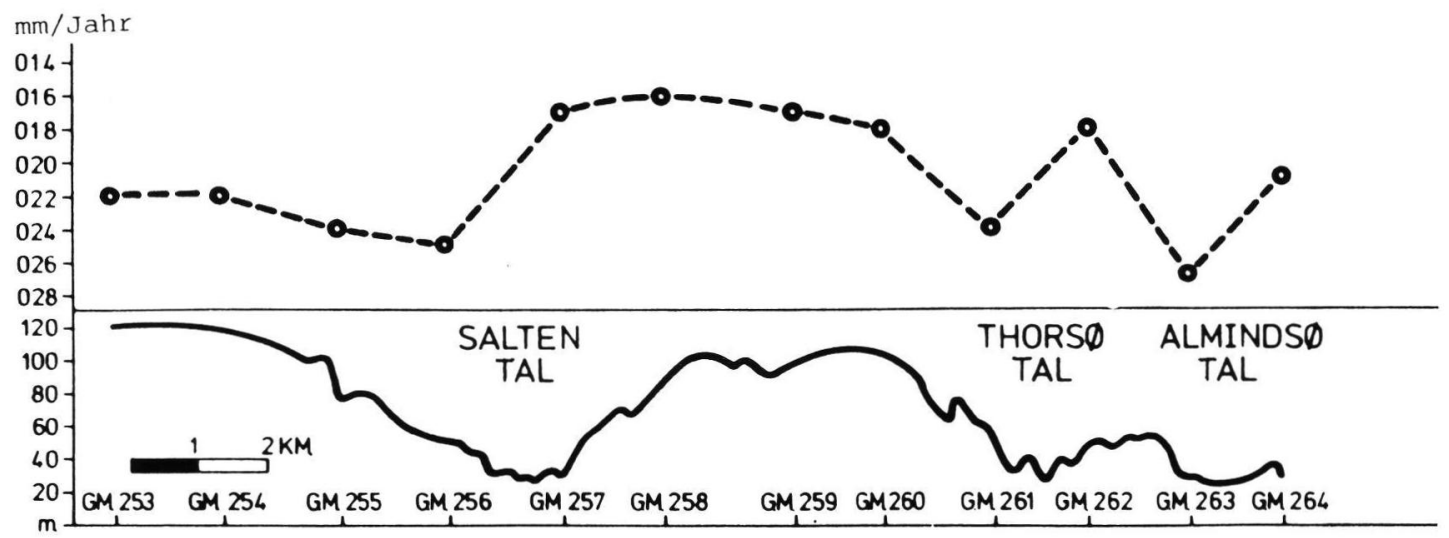

Abb. 16: Darstellung der Senkungsbeträge entlang eines Profils durch Mitteljütland nach KRONBORG (1979). Zur Erläuterung siehe Text: Oben (gestrichelte Linie): Senkung, mm/Jahr, für die Zeit 18911958. Meßstationen im GM-System für Präzisionsnivellements, ausgeführt 1891 und 1958 vom Geodæetisk Institut (heute: Kort- og Matrikelstyrelsen), Kopenhagen. Unten (ausgezogene Linie): Geländeprofil, 25fach überhöht. Zahlen auf der senkrechten Achse: Meter über dem Meeresspiegel.

Fig. 16: Contemporary subsidence of the land surface along a profile through middle Jutland, according to precision levellings made by the Geodætisk Institut, Copenhagen, in the years 1891 and 1958. Full-drawn line, below: landscape section, height scale in meters, 25 times exaggerated as compared to length scale. GM: Points of measuring. Dashed line, above: Subsidence in $\mathrm{mm} / \mathrm{year}$ (note that the largest values are found at the valley sides. 
In Larsen, Kronborg \& Bender (1979) findet sich eine Abbildung (siehe Abb. 16), die zeigt, daß sich die ostjütische Landschaft in den Jahren 1891 bis 1958 gesenkt hat. Die Verfasser weisen darauf hin, daßs sich das Saltenå-Tal stärker als seine Umgebung abgesenkt habe und daß es sich dabei um einen Grabenbruch handeln könne, in dem bis heute eine abwärts gerichtete Bewegung festzustellen sei. Hier liegt aber eine Fehlinterpretation vor: Der Punkt, der am Boden des Saltenå-Tales liegt (GM 257), hat sich mit $18 \mathrm{~mm} /$ Jahr geringer abgesenkt als seine Umgebung, nicht stärker. Die Punkte an den Flanken der drei großen Täler haben sich stärker abgesenkt als alle übrigen Punkte, sowohl an den Talsohlen als auch auf den Höhen. Die einfachste Erklärung hierfür ist, daß es sich um die Auswirkung von Bodenkriechen oder abwärtsgerichtete Bewegung auf Grund des Pflügens handelt, ganz gewöhnliche Hangprozesse, die überall im Gelände festzustellen sind.

Es ist dennoch möglich, daß einige Tunneltäler Bruchlinien im Untergrund oder älteren Schichtgrenzen folgen. Vom Århus-Tal wird angenommen, daß es vor der Saale-Eiszeit angelegt worden ist, möglicherweise schon im Tertiär. Das Tal des Flusses Gudenå folgt auf einer Strecke von ca. 20 km östlich und westlich von Randers der Randsenke um eine Salinarstruktur. Daran ist nichts merkwürdig: Täler können wieder benutzt werden, weil die Erosion dazu neigt, den Linien zu folgen, auf denen bereits Vorarbeit geleistet wurde. So ist wohl bekannt, daß Roskilde-Fjord auf Sjælland einer Bruchlinie folgt. Dennoch zeigt der Verlauf der Oser in seiner Umgebung an, daß die Hohlform auch als Schmelzwassertunnel unter dem Eis des letzten Gletschervorstoßes gewirkt hat.

Die hier vorgeschlagene Interpretation der Tunneltäler bietet gleichzeitig eine Erklärung für eine Reihe von Phänomenen, die vorher schwer zu erklären waren. Beispiele:

a) Die Beobachtung, daß Oser oft an der Sohle von Tunneltälern oder in deren Verlängerung auftreten (Abb. 6 und 15), aber schmaler als diese sind, wurde bereits erwähnt. Das paßt genau zu der neuen Auffassung von der Tunneltalentstehung, wäre ohne diese aber nur schwer zu erklären.

b) Die Tunneltäler, die Nordsjælland etwa in ostwestlicher Richtung in der Umgebung von Farum - Stenløse kreuzen (Abb. 1), scheinen keine Verbindung zu den Ausgangspunkten von Sanderkegeln aufzuweisen. Die oberste Moräne in diesem Gebiet ist von einem Eis ab- gelagert worden, das sich etwa in südsüdostnordnordwestlicher bis süd-nördlicher Richtung bewegt hat, das heißt fast quer zu den Tälern. Die Täler müssen aus dem nächstälteren Eisvorstoß stammen, in dem sich das Eis annähernd ost-westlich bewegt hat. Sie sind erhalten geblieben, weil sie mit Toteis ausgefüllt waren. Eine derartige Verfüllung mit Toteis ist ein integrativer Bestandteil der beschriebenen Tunneltalbildung.

c) Krüger (1989) hebt hervor, daß an Stellen mit einem steilen Eisrand die Äquipotentiallinien dicht geschart liegen. Das Schmelzwasser fließst deshalb schnell und erodiert den Untergrund. Wo das Eis dagegen eine geringe Neigung aufweist, treten große Abstände zwischen den Äquipotentiallinien auf. Das Schmelzwasser wird daher langsam fließen, und die Chancen sind groß, daß es ablagert anstatt zu erodieren. Die Tunneltäler entstehen deshalb in Vorstoßphasen des Eises, die durch einen steilen Eisrand gekennzeichnet sind, während die Oser während der Abschmelzphasen entstehen. Das mitteljütische Eis, d.h. in Deutschland das Eis des Brandenburger/Pommerschen Vorstoßes, das Ussings Tunneltäler hervorgebracht hat, muß während der überwiegenden Zeit seiner Existenz einen recht steilen Eisrand gehabt haben.

d) Infolge der hier beschriebenen TunneltalTheorie wurden die Tunneltäler größer (breiter und tiefer), je länger das Eis an einer bestimmten Stelle liegengeblieben ist. Es sieht so aus, als könne man die Dauer eines Eisvorstoßes aus der Größe der Tunneltäler ableiten. Das ostjütische Eis (vermutlich der RosenthalI-Vorstoß), das infolge neuerer Altersbestimmungen für einige hundert Jahre existiert hat, hat nur wenige und kleine Tunneltäler gebildet. Der mitteljütische Eisvorstoß, der für die Entstehung von Ussings großen Tunneltälern verantwortlich war, hat wahrscheinlich etwa 2000 bis 4000 Jahre gedauert. Große Tunneltäler und große Sanderflächen (wie die westjütischen) gehören zusammen. Ihre Größe verdanken sie kaum Gletscherläufen (jökulhlaups), sondern einer langen Bildungsdauer. Abb. 6 zeigt ein interessantes Beispiel aus Sjælland. Der Verlauf der Eisrandlinien, wie er von Ole Humlum (1976) festgelegt wurde, kann infolgedessen so gedeutet werden, daß das Stadium C kürzere Zeit gedauert hat als A bis B. Das kleine Pottesø-Klarsø-Kragsø-Tal nordöstlich von Ringsted ist ein typisches Beispiel für 
ein Tunneltal, das an der Eisrandlinie $\mathrm{C}$ endet. Wir haben es hier mit einem Tal zu tun, das in wenigen Jahren von einem Wasserstrom erzeugt wurde, der seinen Lauf vielleicht nicht verlagert hat. Das Tal ist von derselben Größenordnung wie die meisten Oser.

e) Aabenaa Fjord (Abb. 17) ist ein Sonderfall eines jütischen Fjords, der sowohl sehr breit als auch tief ist, und dessen inneres Ende von einer zungenförmigen Eisrandlinie umgeben ist. Fast alle Tunneltäler münden im Gegensatz zu diesem Tal in einer Kerbe im Eisrand (siehe Abb. 1, 6, 13). Wenn diese Förde als Tunneltal angesehen werden soll, müssen diese Abweichungen auf besondere Bedingungen zurückzuführen sein. Nun weiß man inzwischen, daß das Gebiet um Aabenraa in der letzten Eis-

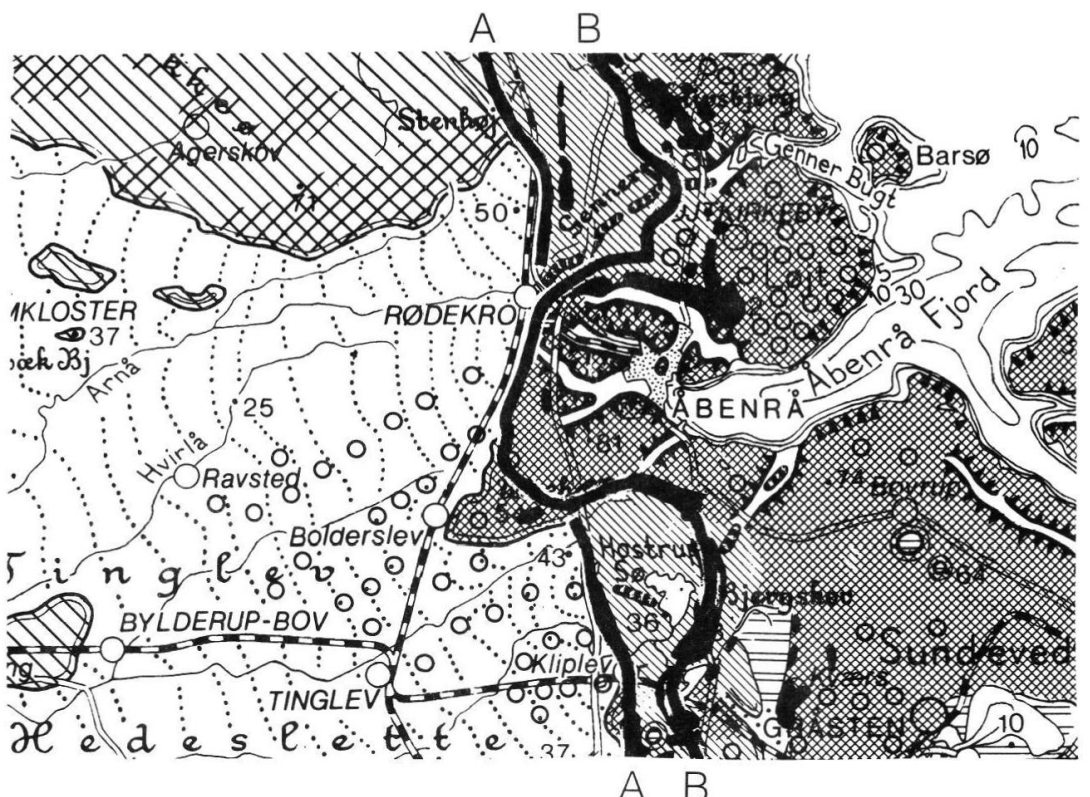

Zeichenerklärung:
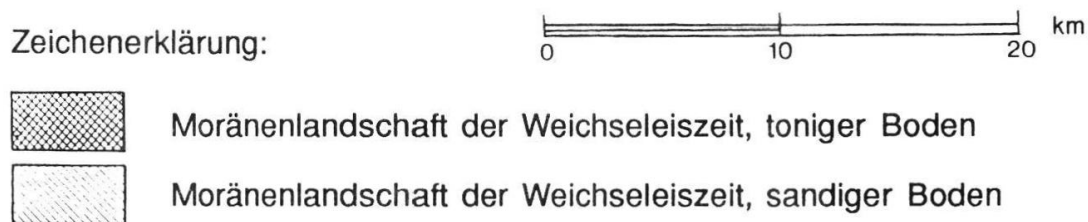

Moränenlandschaft der Weichseleiszeit, toniger Boden

Moränenlandschaft der Weichseleiszeit, sandiger Boden

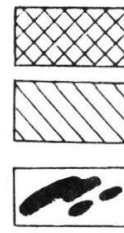

Altmoräne, toniger Boden

Altmoräne, sandiger Boden

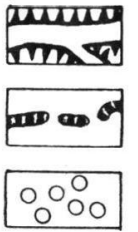

Tunneltäler

Endmoränenlandschaft

Oser

$\because \because \ddots \quad$ Sanderflächen

Toteisrelief

Abb. 17: Aabenraa Fjord und Umgebung. Ausschnitt aus Per Smeds „Landskabskort over Danmark, blad nr.3 (Geografforlaget)“. A = mitteljütische Eisrandlinie; $\mathrm{B}=$ ostjütische Eisrandlinie.

Fig. 17: Aabenraa fjord (inlet) and vicinity, section of "Landskabskort over Danmark", sheet 3 (SMED). A = Mid Jutland ice margin line. B = East Jutland ice margin line. Explanation of signs, from above: Weichselian moraine landscape, clayey; the same, sandy; Saalian moraine landscape, clayey; the same, sandy; end moraines; sandurs; tunnel valleys; eskers; dead-ice relief.

zeit zweimal vom Eis bedeckt war, zum ersten Mal während des mitteljütischen (pommerschen) Vorstoßes (Ussings Eisvorstoß), und danach während des ostjütischen („Rosenthal")Eisvorstoßes - und daß der zungenförmige Vorsprung der Eisrandlinie zu dem jüngsten, also dem ostjütischen Eisvorstoß gehört (HANSEN 1978). Westlich der Stadt Aabenraa finden sich mehrere kleine Tunneltäler, die fächerförmig auf den Rand der Eiszunge zulaufen; sie sind unter dem ostjütischen Eis gebildet worden. Die tiefe Förde, die nordost-südwestlich streicht, ist offenbar schon vorher angelegt gewesen. Die Förde könnte ursprünglich ein Tunneltal des mitteljütischen Eisvorstoßes gewesen sein; der ostjütische Vorstoß hat nur seine Form modifiziert. Das ostjütische Eis hat sich in ähnlicher Weise auch in Nordjütland verhalten, mit Zungen, die in offenbar schon vorher vorhandene Niederungen vorgestoßen 
sind; keine der Zungen sind jedoch mehr als halb so lang wie breit (siehe Abb. 1).

Es sei daran erinnert, daß für GRIPP (1964) Aabenraa Fjord einer der Anstöße gewesen ist, die die (falsche) Gletscherzungentheorie ausgelöst haben. Kurioserweise müssen wir in diesem speziellen Fall Gripp teilweise Recht geben; aber dies ist ein Sonderfall, der nicht verallgemeinert werden kann. Aabenraa Fjord ist ein Mittelding zwischen einem Tunneltal und einem Zungenbecken. Ein Verständnis dessen, was hier vorgegangen ist, setzt eine klare Vorstellung der beiden Komponenten voraus, die an der Entstehung beteiligt waren. Möge dieser Artikel hierzu einen Beitrag leisten.

\section{Danksagung}

Dieser Artikel hätte nicht ohne die Hilfe von Ole Humlum, Glaziologe am Geografisk Institut, Universität von Kopenhagen, geschrieben werden können. Humlums Geduld damit, komplizierte Zusammenhänge zu erklären, ist fast einzigartig. Zeitmangel hat ihn daran gehindert, diesen Artikel selbst zu schreiben. Jürgen EHLERs, Hamburg, hat das Manuskript ins Deutsche übersetzt. Beiden möchte ich für ihre Mühe danken.

\section{Schriftenverzeichnis}

EhlERs, J. (1994): Allgemeine und historische Quartärgeologie. Enke, Stuttgart. 358 S., 176 Abb.

- \& LinKe, G. (1989): The origin of deep buried channels of Elsterian age in Northwest Germany. Journal of Quaternary Science, $\mathbf{4}:$ 255-265.

- \& Wingfeld, R. (1991): The extension of the Late Weichselian/Late Devensian ice sheets in the North Sea Basin. Journal of Quaternary Science, 6: 313326.

Franz, H.-J. (1965): Die Geomorphologie des Jungmoränengebietes, in: Gellert, J. F. (Hrsg.): Die Weichsel-Eiszeit im Gebiet der Deutschen Demokratischen Republik: 212-229. Berlin: AkademieVerlag.

GRIPP, K. (1964): Erdgeschichte von Schleswig-Holstein. Karl Wachholz-Verlag, Neumünster. 411 p., 57 Tafeln.

Grube, F. (1979): Übertiefte Täler im Hamburger Raum. Eiszeitalter und Gegenwart, 29: 157-172.

Hansen, K. (1971): Tunnel valleys in Denmark and Northern Germany. Bulletin of the Geological Society of Denmark, 20: 295-306.
Hansen, S. (1978): Sidste nedisnings maksimumsudbredelse i Syd- og Midtjylland. DGU, Årbog 1976: 139-152.

Humlum, O. (1976): Sorø- og Stenlilleegnens geomorfologi. Unpublished report, Geographical Institute, Univ. of Copenhagen, $383 \mathrm{pp}$.

KrÜGer, J. (1989): Gletscheren og landskabet. 77 pp. København (Gyldendal).

Kuster, H. \& Meyer, K.-D. (1979): Glaziäre Rinnen im mittleren und nordöstlichen Niedersachsen. Eiszeitalter und Gegenwart, 29: 135-156.

Larsen, G. \& Kronborg, C. (1994): Det mellemste Jylland; Geologisk set. Skov- og Naturstyrelsen/Geografforlaget. 272 pp.

Larsen, G., Kronborg, C. \& Bender, H. (1979): Det midtjyske Søhøjland, Geologi. Århus amtskommune, Amtsfredningskontoret. 31 pp.

LyKKe-ANDERSEN, H. (1986): On the buried Nørreå valley. Geoskrifter, 24: 211-223.

- (1995): Om tunneldalenes natur. Geologisk Nyt (3): 17-19.

NyE, J. F. (1952): The mechanics of glacier flow. Journal of Glaciology, 2: 82-93.

RüHBERG, N. (1987): Die Grundmoräne des jüngsten Weichselvorstoßes im Gebiet der DDR. Zeitschrift für Geologische Wissenschaften, 15: 759-767.

ScHulz, W. (1967): Abriß der Quartärgeologie Mecklenburgs. Archiv der Freunde der Naturgeschichte Mecklenburgs, XIII: 99-119.

Shreve, R. L. (1972): Movement of water in glaciers. Journal of Glaciology, 11: 205-214.

SMED, P. (1995): Tunneldale - er dannet af smeltevand under isen. Geologisk Nyt (1): 19-23, (2): 19-23.

Sugden, D.E. \& John, B. S. (1979): Glaciers and landscape. 376 pp. London (Arnold).

Ussing, N. V. (1903): Om Jyllands Hedesletter og Teorierne for deres Dannelse. Det Kgl. Danske Videnskabernes Selskabs Forhandlinger, 2: 99-164.

- (1907): Om Floddale og Randmoræner i Jylland. Det Kgl. Danske Videnskabernes Selskabs Forhandlinger, 4: 162-214.

VARV (1992): Geologisk kort over den danske undergrund, red. E. HÁKAnsSon \& S.S. PEDERSEN.

Woldstedt, P. (1950): Norddeutschland und angrenzende Gebiete im Eiszeitalter. 464 S. Stuttgart (Koehler).

Woldstedt, P. \& Duphorn, K. (1974): Norddeutschland und angrenzende Gebiete im Eiszeitalter, 3. Auflage. 500 S. Stuttgart (Koehler).

Manuskript eingegangen am 6. Januar 1997 